\title{
TANNAKIAN CATEGORIES, LINEAR DIFFERENTIAL ALGEBRAIC GROUPS, AND PARAMETERIZED LINEAR DIFFERENTIAL EQUATIONS
}

\author{
ALEXEY OVCHINNIKOV
}

\begin{abstract}
We provide conditions for a category with a fiber functor to be equivalent to the category of representations of a linear differential algebraic group. This generalizes the notion of a neutral Tannakian category used to characterize the category of representations of a linear algebraic group [18, 9].
\end{abstract}

\section{INTRODUCTION}

Tannaka's Theorem (c.f., [19]) states that a linear algebraic group is determined by its category of representations. The problem of recognizing when a category is the category of representations of a linear algebraic group (or more generally, an affine group scheme) is attacked via the theory of neutral Tannakian categories (see $[18,9])$. This theory allows one to detect the underlying presence of a linear algebraic group in various settings. For example, the Galois theory of linear differential equations can be developed in this context (see [9, 10, 15]).

In [2], Cassidy introduced the concept of a differential algebraic group and in [3] studied the representation theory of linear differential algebraic groups. Building on this work, we proved an analogue of Tannaka's Theorem for linear differential algebraic groups (see [17]). In the present paper, we develop the notion of a neutral differential Tannakian category and show that this plays the same role for linear differential algebraic groups that the theory of neutral Tannakian categories plays for linear algebraic groups. As an application, we are able to give a categorical development of the theory of parameterized linear differential equations that was introduced in [4].

Another approach to the Galois theory of systems of linear differential equations with parameters is given in [1], where the authors study Galois groups for generic values of the parameters. Also, it is shown in [8] that over the field $\mathbb{C}(x)$ of rational functions over the complex numbers the differential Galois group of a parameterized system of differential equations will be the same for all values of the parameter outside a countable union of proper algebraic sets.

The way we define differential Tannakian categories here relies on existence of a fiber functor that "commutes" with the differential structure. It turns out that there is a treatment of differential Tannakian categories not based on the fiber functor [16], where there given conditions for a rigid abelian tensor category with an

Date: September 20, 2018.

1991 Mathematics Subject Classification. 12H05, 57T05, 18E99.

Key words and phrases. Tannakian categories, linear differential equations, Galois groups.

The work was partially supported by NSF Grant CCR-0096842 and by the Russian Foundation for Basic Research, project no. 05-01-00671. 
additional differential structure so that the category has a fiber functor compatible with the differential structure. This is done based on the ideas of this paper, in the spirit of [10], and extending [11].

The paper is organized as follows. In Sections 2 and 3 we review the basic properties of linear differential algebraic groups and define and develop basic properties of a category which is a differential analogue of a neutral Tannakian category. In Section 4 we prove the main result of the paper: namely, there is a (pro-)linear differential algebraic group such that a given neutral differential tannakian category is the category of representations of the group. We give an application of this result to the theory of parameterized linear differential equations in Section 5. The techniques we use are built on the techniques introduced in $[18,9]$ but the presence of the differential structure introduces new subtleties and several new constructions.

\section{BAsiC Definitions}

A $\Delta$-ring $R$, where $\Delta=\left\{\partial_{1}, \ldots, \partial_{m}\right\}$, is a commutative associative ring with unit 1 and commuting derivations $\partial_{i}: R \rightarrow R$ such that

$$
\partial_{i}(a+b)=\partial_{i}(a)+\partial_{i}(b), \quad \partial_{i}(a b)=\partial_{i}(a) b+a \partial_{i}(b)
$$

for all $a, b \in R$. If $\mathbf{k}_{\mathbf{0}}$ is a field and a $\Delta$-ring then $\mathbf{k}_{\mathbf{0}}$ is called a $\Delta$-field. We restrict ourselves to the case of

$$
\text { char } \mathbf{k}_{\mathbf{0}}=0 .
$$

If $\Delta=\{\partial\}$ then we call a $\Delta$-field as $\partial$-field. For example, $\mathbb{Q}$ is a $\partial$-field with the unique possible derivation (which is the zero one). The field $\mathbb{C}(t)$ is also a $\partial$-field with $\partial(t)=f$, and this $f$ can be any rational function in $\mathbb{C}(t)$. Let $C$ be the field of constants of $\mathbf{k}_{\mathbf{0}}$, that is, $C=\operatorname{ker} \partial$.

In Section 5 we require that every consistent system of algebraic differential equations (that is, it has a solution in an extension of $\mathbf{k}_{\mathbf{0}}$ ) with coefficients in $\mathbf{k}_{\mathbf{0}}$ has a solution in $\mathbf{k}_{\mathbf{0}}$. Such a field is called differentially closed. In characteristic zero for a $\Delta$-field one can construct its differential algebraic closure unique up to an isomorphism (see [4, Definition 3.2] and the references given there). Also, in Section 5 we will deal with a field $\mathbf{k}$ equipped with two commuting differentiations, $\partial_{x}$ and $\partial_{t}$, and $\mathbf{k}_{\mathbf{0}}$ is the $\partial_{t}$-field of $\partial_{x}$-constants of $\mathbf{k}$ and the differentiation $\partial_{t}$ that we use in Section 5 plays the role of $\partial$ that we have here.

Let

$$
\Theta=\left\{\partial^{i} \mid i \in \mathbb{Z}_{\geqslant 0}\right\}
$$

Since $\partial$ acts on a $\partial$-ring $R$, there is a natural action of $\Theta$ on $R$. A non-commutative $\operatorname{ring} R[\partial]$ of linear differential operators is generated as a left $R$-module by the monoid $\Theta$. A typical element of $R[\partial]$ is a polynomial

$$
D=\sum_{i=1}^{n} a_{i} \partial^{i}, a_{i} \in R .
$$

The right $R$-module structure follows from the formula

$$
\partial \cdot a=a \cdot \partial+\partial(a)
$$

for all $a \in R$. We denote the set of operators in $R[\partial]$ of order less than or equal to $p$ by $R[\partial] \leqslant p$.

Let $R$ be a $\partial$-ring. If $B$ is an $R$-algebra, then $B$ is a $\partial$-R-algebra if the action of $\partial$ on $B$ extends the action of $\partial$ on $R$. If $R_{1}$ and $R_{2}$ are $\partial$-rings then a ring 
homomorphism $\varphi: R_{1} \rightarrow R_{2}$ is called a $\partial$-homomorphism if it commutes with $\partial$, that is,

$$
\varphi \circ \partial=\partial \circ \varphi
$$

We denote these homomorphisms simply by $\operatorname{Hom}\left(R_{1}, R_{2}\right)$. If $A_{1}$ and $A_{2}$ are $\partial-$ $\mathbf{k}_{\mathbf{0}}$-algebras then a $\partial-\mathbf{k}_{\mathbf{0}}$-homomorhism simply means a $\mathbf{k}_{\mathbf{0}}[\partial]$-homomorphism. We denote the category of $\partial-\mathbf{k}_{\mathbf{0}}$-algebras by $\mathbf{A l g}_{\mathbf{k}_{\mathbf{0}}}(\partial)$. Let $Y=\left\{y_{1}, \ldots, y_{n}\right\}$ be a set of variables. We differentiate them:

$$
\Theta Y:=\left\{\partial^{i} y_{j} \mid i \in \mathbb{Z}_{\geqslant 0}, 1 \leqslant j \leqslant n\right\} .
$$

The ring of differential polynomials $R\{Y\}$ in differential indeterminates $Y$ over a $\partial$-ring $R$ is the ring of commutative polynomials $R[\Theta Y]$ in infinitely many algebraically independent variables $\Theta Y$ with the differentiation $\partial$, which naturally extends $\partial$-action on $R$ as follows:

$$
\partial\left(\partial^{i} y_{j}\right):=\partial^{i+1} y_{j}
$$

for all $i \in \mathbb{Z}_{\geqslant 0}$ and $1 \leqslant j \leqslant n$. A $\partial$ - $\mathbf{k}_{0}$-algebra $A$ is called finitely $\partial$-generated over $\mathbf{k}_{\mathbf{0}}$ if there exists a finite subset $X=\left\{x_{1}, \ldots, x_{n}\right\} \subset A$ such that $A$ is a $\mathbf{k}_{\mathbf{0}}$-algebra generated by $\Theta X$.

An ideal $I$ in a $\partial$-ring $R$ is called differential if it is stable under the action of $\partial$, that is,

$$
\partial(a) \in I
$$

for all $a \in I$. If $F \subset R$ then $[F]$ denotes the differential ideal generated by $F$. If a differential ideal is radical, it is called radical differential ideal. The radical differential ideal generated by $F$ is denoted by $\{F\}$. If a differential ideal is prime, it is called a prime differential ideal.

Definition 1 . The category $\mathcal{V}$ over a $\partial$-field $\mathbf{k}_{\mathbf{0}}$ is the category of finite dimensional vector spaces over $\mathbf{k}_{\mathbf{0}}$ :

(1) objects are finite dimensional $\mathbf{k}_{\mathbf{0}}$-vector spaces,

(2) morphisms are $\mathbf{k}_{\mathbf{0}}$-linear maps;

with tensor product $\otimes$, direct $\operatorname{sum} \oplus$, dual $*$, and additional operations:

$$
F^{p}: V \mapsto V^{(p)}:=\mathbf{k}_{\mathbf{0}}[\partial]_{\leqslant p} \otimes V,
$$

which we call differentiation (or prolongation) functors. If $\varphi \in \operatorname{Hom}(V, W)$ then we define

$$
F^{p}(\varphi): V^{(p)} \rightarrow W^{(p)}, \varphi\left(\partial^{q} \otimes v\right)=\partial^{q} \otimes \varphi(v), 0 \leqslant q \leqslant p .
$$

Here, $\mathbf{k}_{\mathbf{0}}[\partial]_{\leqslant p}$ is considered as the right $\mathbf{k}_{\mathbf{0}}$-module of differential operators up to order $p$ and $V$ is viewed as a left $\mathbf{k}_{\mathbf{0}}$-module. We denote $F^{1}$ simply by $F$.

For each $V \in \mathcal{O b}(\mathcal{V})$ there are: a natural inclusion

$$
i: V \rightarrow V^{(1)}, \quad v \mapsto 1 \otimes v, v \in V,
$$

a "derivation"

$$
\partial: V \rightarrow V^{(1)}, \quad v \mapsto \partial \otimes v,
$$

and a projection

$$
\varphi: V^{(1)} \rightarrow V, \quad 1 \otimes v \mapsto 0, \partial \otimes v \mapsto v .
$$

Remark 1. If $\left\{v_{1}, \ldots, v_{n}\right\}$ is a basis of $V$ then $\left\{v_{1}, \ldots, v_{n}, \ldots, \partial^{p} \otimes v_{1}, \ldots, \partial^{p} \otimes v_{n}\right\}$ is a basis of $V^{(p)}$. 


\section{General Definition of a Differential Tannakian CAtegory}

\subsection{Definition.}

Definition 2. A neutral differential Tannakian category $\mathcal{C}$ over a $\partial$-field $\mathbf{k}_{\mathbf{0}}$ of characteristic zero is a

(1) rigid

(2) abelian

(3) tensor

category such that $\mathbf{E n d}(\underline{1})$ is the field $\mathbf{k}_{\mathbf{0}}$ supplied with an

(1) exact

(2) faithful

(3) $\mathbf{k}_{\mathbf{0}}$-linear

(4) tensor

fiber functor $\omega: \mathcal{C} \rightarrow \mathcal{V}$ (see Definition 1); with a functor

$$
F: X \mapsto X^{(1)},
$$

intertwining $^{1}$ with $\omega$ :

$$
F_{\mathcal{V}} \circ \omega=\omega \circ F_{\mathcal{C}}
$$

where the subscripts $\mathcal{C}$ and $\mathcal{V}$ indicate on which category the functor $F$ acts $^{2}$. Also, for each $X \in \mathcal{O b}(\mathcal{C})$ there must be an inclusion (a morphism with the trivial kernel)

$$
i: X \rightarrow X^{(1)}
$$

and surjection

$$
\phi: X^{(1)} \rightarrow X
$$

morphisms in $\mathcal{C}$, so that the sequence

$$
0 \longrightarrow X \stackrel{i}{\longrightarrow} X^{(1)} \stackrel{\phi}{\longrightarrow} X \longrightarrow 0
$$

is exact and is mapped by the fiber functor $\omega$ to the corresponding exact sequence in the category $\mathcal{V}$ :

$$
0 \longrightarrow \omega(X) \stackrel{i}{\longrightarrow} \omega(X)^{(1)} \stackrel{\varphi}{\longrightarrow} \omega(X) \longrightarrow 0,
$$

where the $\mathbf{k}_{\mathbf{0}}$-linear maps $i$ and $\varphi$ are defined in (1) and (3), respectively. Moreover, we require that the differential structure respects tensor products ("product rule"), that is, for the natural $\mathbf{k}_{\mathbf{0}}$-linear map

$$
(\omega(X) \otimes \omega(Y))^{(1)} \rightarrow \omega(X)^{(1)} \otimes \omega(Y)^{(1)}
$$

mapping

$$
\begin{aligned}
1 \otimes(v \otimes u) & \mapsto(1 \otimes v) \otimes(1 \otimes u), \\
\partial \otimes(v \otimes u) & \mapsto(\partial \otimes v) \otimes(1 \otimes u)+(1 \otimes v) \otimes(\partial \otimes u)
\end{aligned}
$$

there exists a corresponding morphism in the category $\mathcal{C}$

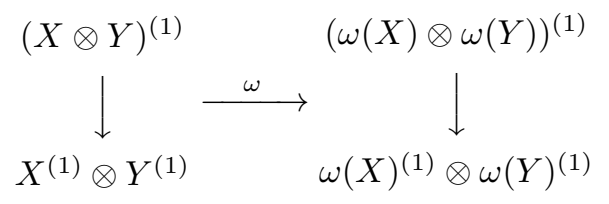

\footnotetext{
${ }^{1}$ This means that there is a functorial in $X$ isomorphism $\omega\left(X^{(1)}\right) \cong \omega(X)^{(1)}$.

${ }^{2}$ In Section 5 we will make use of $F^{p}$ on $\mathcal{V}$ for $p>1$ for computational purposes.
} 
Also, we require that there exists a morphism $g_{X}:\left(X^{*}\right)^{(1)} \rightarrow X^{(1)^{*}}$ satisfying the following commutative diagram:

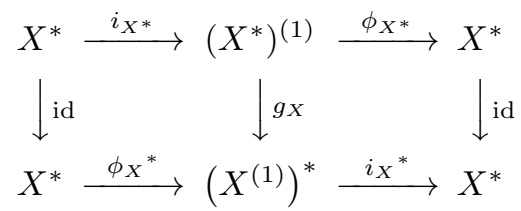

such that the $\mathbf{k}_{\mathbf{0}}$-linear isomorphism $\omega\left(g_{X}\right): \omega\left(X^{*}\right)^{(1)} \rightarrow \omega\left(X^{(1)}\right)^{*}$ is of the form:

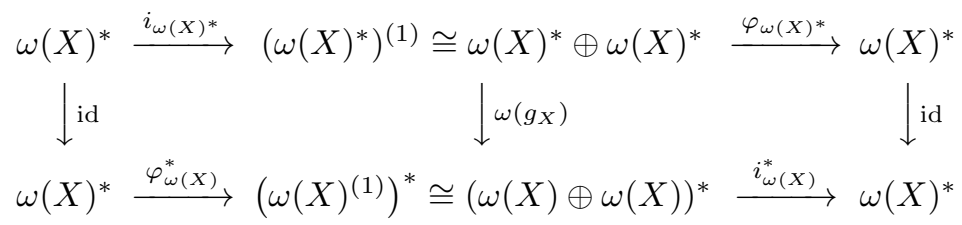

with the map $\omega\left(g_{X}\right)$ respecting the given splittings into direct sums.

In Example 5, Section 3.3, we will show that the category of differential representations of a linear differential algebraic group together with the forgetful functor form a neutral differential Tannakian category.

Remark 2. The functor $F$ and these additional morphisms are used directly to recover the differential structure on the (pro-)linear differential algebraic group whose category of representations is $\mathcal{C}$. This explains why they are given here. In particular, conditions (6) and (7) are given to ensure that the coinverse on the Hopf algebra that we recover is a differential homomorphism. This will further be discussed in Lemma 6 and Proposition 2.

Our main goal is to prove the following theorem.

Theorem 1. For a neutral differential Tannakian category $\mathcal{C}$ :

- the functor $\mathbf{A u t}^{\otimes, \partial}(\omega)$ from $\mathbf{A l g}_{\mathbf{k}_{\mathbf{0}}}(\partial)$ to $\{$ Groups\} is representable by a differential Hopf algebra A,

- the functor $\omega$ defines an equivalence of tensor categories $\mathcal{C} \rightarrow \mathbf{R e p}_{G}$, with $G$ being the affine differential group scheme represented by $A$.

We start with developing a technique for this. We partially follow $[9$, pages 130-137] modifying their definitions and proofs to give the correct result in the differential case. In Example 6, Section 3.5, we will show the "easy part" of Theorem 1 when $\mathcal{C}=\operatorname{Rep}_{G}$.

3.2. Tensor product $\mathcal{C} \otimes \mathcal{V}$ and its properties. In this section we will list several lemmas given in [9, pages 131-132] that we need to use. Let $(\mathcal{C}, \omega)$ be a neutral differential Tannakian category. Consider objects $V \in \mathcal{O b}(\mathcal{V})$ and $X \in \mathcal{O b}(\mathcal{C})$.

Definition 3. We define

$$
V \otimes X=\left\{\left[\left(X^{n}\right)_{\alpha}, \phi_{\beta, \alpha}\right] \mid \alpha:\left(\mathbf{k}_{\mathbf{0}}{ }^{n}\right) \cong V\right\},
$$

where $\left(X^{n}\right)_{\alpha}:=X \oplus \ldots \oplus X$ and $\phi_{\beta, \alpha}:\left(X^{n}\right)_{\alpha} \rightarrow\left(X^{n}\right)_{\beta}$ is defined by $\beta^{-1} \circ \alpha$ acting as an element of $\mathbf{G L}_{n}\left(\mathbf{k}_{\mathbf{0}}\right)$.

Lemma 1. $\phi_{\gamma, \beta} \circ \phi_{\beta, \alpha}=\phi_{\gamma, \alpha}$. 
Definition 4. For an object $T \in \mathcal{O b}(\mathcal{C})$ we say that $\Phi \in \operatorname{Hom}(V \otimes X, T)$ if $\Phi=\left\{\varphi_{\alpha}:\left(X^{n}\right)_{\alpha} \rightarrow T\right\}$ making possible diagrams with $\phi_{\beta, \gamma}$ commutative. Also, $\Phi \in \operatorname{Hom}(T, V \otimes X)$ if $\Phi=\left\{\left(\varphi_{1}, \ldots, \varphi_{n}\right)_{\alpha}\right\}, \varphi_{i}: T \rightarrow X$ making possible diagrams with $\phi_{\beta, \gamma}$ commutative.

Recall that $\mathbf{k}_{\mathbf{0}} \subset \operatorname{End}(X)$.

Lemma 2. For $V \in \mathcal{O b}(\mathcal{V})$ there is a canonical $\mathbf{k}_{\mathbf{0}}$-linear map

$$
\begin{gathered}
V \rightarrow \operatorname{Hom}(X, V \otimes X), \quad v \mapsto\left(\left(\varphi^{1}, \ldots, \varphi^{n}\right)_{\alpha}: X \rightarrow X_{\alpha}\right), \\
\left(\varphi^{i}\right)_{\alpha} \text { is the multiplication by } w^{i},
\end{gathered}
$$

where $\alpha^{-1}(v)=w, w=\left(w^{1}, \ldots, w^{n}\right) \in \mathbf{k}_{\mathbf{0}}{ }^{n}$.

Lemma 3. We have a functorial isomorphism

$$
\operatorname{Hom}(V \otimes X, T) \cong \operatorname{Hom}(V, \operatorname{Hom}(X, T)),
$$

where $T$ is an object in $\mathcal{C}$.

Lemma 4. Let $F: \mathcal{C} \rightarrow \mathcal{C}^{\prime}$ be a functor. Then $F(V \otimes X)=V \otimes F(X)$.

Lemma 5. Let $\mathcal{C}=\mathcal{V}$ then $V \otimes X \cong V \otimes \mathcal{V} X$ (the tensor product in $\mathcal{V})$.

3.3. Differential comodules. Let $A$ be a $\partial-\mathbf{k}_{\mathbf{0}}$-algebra. Assume that $A$ is supplied with the following operations:

- differential algebra homomorphism $m: A \otimes A \rightarrow A$ is the multiplication map on $A$,

- differential algebra homomorphism $\Delta: A \rightarrow A \otimes A$ which is a comultiplication,

- differential algebra homomorphism $\varepsilon: A \rightarrow \mathbf{k}_{\mathbf{0}}$ which is a counit,

- differential algebra homomorphism $S: A \rightarrow A$ which is a coinverse.

We also assume that these maps satisfy commutative diagrams (see [3, page 225]):

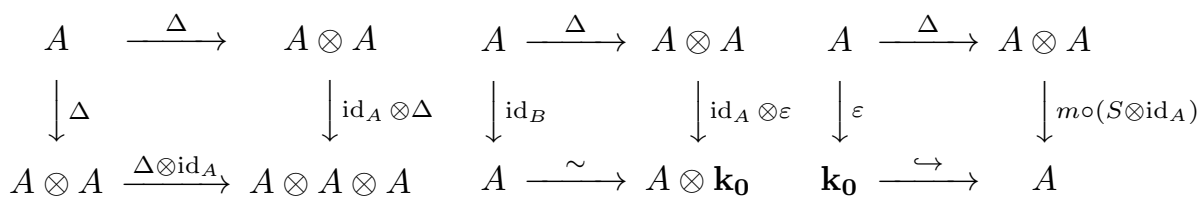

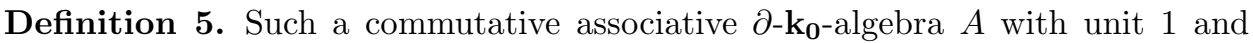
operations $m, \Delta, S$, and $\varepsilon$ satisfying axioms (8) is called a differential Hopf algebra (or $\partial$ - $\mathbf{k}_{\mathbf{0}}$-Hopf algebra).

Definition 6. Assume that $A$ is just a vector space over $\mathbf{k}_{\mathbf{0}}$ with a derivation $\partial$ which extends the derivation on $\mathbf{k}_{\mathbf{0}}$ equipped with $\Delta$ and $\varepsilon$ commuting with $\partial$. In this case $A$ is called a differential coalgebra. When, in addition, $A$ has $S$, it is called a differential bialgebra.

Definition 7. A finite dimensional vector space $V$ over $\mathbf{k}_{\mathbf{0}}$ is called a $A$-comodule if there is a given $\mathbf{k}_{\mathbf{0}}$-linear morphism

$$
\rho: V \rightarrow V \otimes A,
$$


satisfying the axioms:

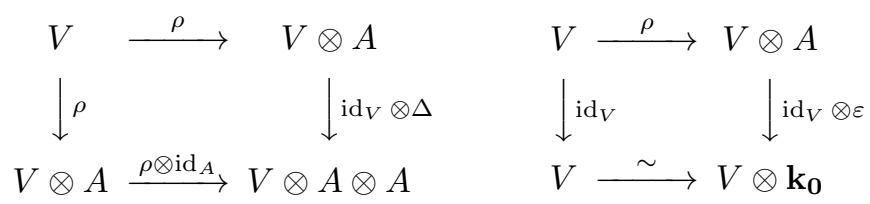

If $A$ is a differential coalgebra with $\Delta$ and $\varepsilon$ then for a comodule $V$ over $A$ the $\mathbf{k}_{\mathbf{0}}$ space $\mathbf{k}_{\mathbf{0}}[\partial]_{\leqslant i} \otimes V=V^{(i)}$ has a natural $A$-comodule structure

$$
\rho(f \otimes v):=f \otimes \rho(v)
$$

for $f \in \mathbf{k}_{\mathbf{0}}[\partial]_{\leqslant i}$ and $v \in V$.

We denote the category of comodules over a differential coalgebra $A$ by $\operatorname{CoDiff}_{A}$ with the induced differentiation (9). For a $\partial-\mathbf{k}_{\mathbf{0}}$-Hopf algebra $A$ the functor

$$
G: \mathbf{A l g}_{\mathbf{k}_{\mathbf{0}}}(\partial) \rightarrow\{\text { Groups }\}, \quad R \mapsto \operatorname{Hom}(A, R)
$$

is called an affine differential algebraic group scheme generated by $A$ (see [17, Section 3.3]). In this case $V \in \mathcal{O b}\left(\mathbf{C o D i f f}{ }_{A}\right)$ is called a differential representation of $G$ ([17, Definition 7, Theorem 1]). The category $\mathbf{C o D i f f}_{A}$ is also denoted by $\mathbf{R e p}_{G}$.

The differential $\mathbf{G L}_{n}$ by definition is the functor represented by the $\partial-\mathbf{k}_{\mathbf{0}}-\mathrm{Hopf}$ algebra

$$
\mathbf{k}_{0}\left\{X_{11}, \ldots, X_{n n}, 1 / \operatorname{det}(X)\right\},
$$

where $X_{i j}$ are differential indeterminates. The comultiplication $\Delta$ and coinverse $S$ are defined on $X_{i j}$ in the usual way. Their prolongation on the derivatives of $X_{i j}$ can be obtained by differentiation.

Example 1. Denote the differential $\mathbf{G L}_{1}$ by $\mathbf{G}_{\mathbf{m}}$. The $\partial-\mathbf{k}_{\mathbf{0}}$-Hopf algebra for $\mathbf{G}_{\mathbf{m}}$ is then $\mathbf{k}_{\mathbf{0}}\{y, 1 / y\}$. We have:

$$
\begin{aligned}
& \Delta(y)=y \otimes y, \\
& S(y)=1 / y .
\end{aligned}
$$

These maps are $\partial$-homomorphisms. Therefore,

$$
\begin{aligned}
& \Delta(\partial y)=\partial(\Delta(y))=\partial y \otimes y+y \otimes \partial y, \\
& S(\partial y)=\partial(S(y))=\partial(1 / y)=-\partial y / y^{2}
\end{aligned}
$$

and so on.

Recall that $C$ is the field of $\partial$-constants of $\mathbf{k}_{\mathbf{0}}$.

Example 2. The "constant" multiplicative group $\mathbf{G}_{\mathbf{m}}(C)$ is the subgroup of $\mathbf{G}_{\mathbf{m}}$ given by the equation $\partial y=0$. So, the $\partial-\mathbf{k}_{\mathbf{0}}$-Hopf algebra of $\mathbf{G}_{\mathbf{m}}(C)$ is

$$
\mathbf{k}_{\mathbf{0}}\{y, 1 / y\} /[\partial y] \cong \mathbf{k}_{\mathbf{0}}[y, 1 / y]
$$

which is the usual algebraic multiplicative group.

Example 3. The differential $\mathbf{G}_{\mathbf{m}}$ has another non-trivial differential algebraic subgroup given by the following differential equation

$$
\partial\left(\frac{\partial y}{y}\right)=0
$$

(see $[4$, page 126] for more information). 
Example 4. The differential $\mathbf{G}_{\mathbf{a}}$ is represented by the $\partial-\mathbf{k}_{\mathbf{0}}$-Hopf algebra $\mathbf{k}_{\mathbf{0}}\{y\}$ with

$$
\begin{aligned}
\Delta\left(\partial^{p} y\right) & =\partial^{p} y \otimes 1+1 \otimes \partial^{p} y, \\
S\left(\partial^{p} y\right) & =(-1)^{p+1} \partial^{p} y
\end{aligned}
$$

for all $p \in \mathbb{Z}_{\geqslant 0}$.

Definition 8. An affine differential algebraic group scheme $G$ is called a linear differential algebraic group if there exists an imbedding:

$$
G \rightarrow \mathbf{G L}_{n}
$$

for some $n \in \mathbb{Z}_{\geqslant 1}$.

Example 5. We show that for a linear differential algebraic group $G$ the category $\mathbf{R e p}_{G}$ together with the forgetful functor form a neutral differential Tannakian category. Indeed, in the above it is shown how to define the functor $F$. Moreover, for any $g \in G, V$ and $W \in \mathcal{O b}\left(\operatorname{Rep}_{G}\right), v \in V, w \in W$ we have:

$$
V \ni g \cdot v \mapsto 1 \otimes(g \cdot v)=g \cdot(1 \otimes v) \in V^{(1)} ;
$$

in addition, applying $\partial: V \rightarrow V^{(1)}$ we obtain

$$
g \cdot v \mapsto \partial \otimes(g \cdot v)=g \cdot(\partial \otimes v)
$$

also, for the product rule we have:

$$
\begin{aligned}
(V \otimes W)^{(1)} \ni & g \cdot(\partial \otimes(v \otimes w))=\partial \otimes(g \cdot v \otimes g \cdot w) \mapsto \\
& \mapsto(\partial \otimes g \cdot v) \otimes(1 \otimes g \cdot w)+(1 \otimes g \cdot v) \otimes(\partial \otimes g \cdot w)= \\
& =g \cdot((\partial \otimes v) \otimes(1 \otimes w)+(1 \otimes v) \otimes(\partial \otimes w)) \in V^{(1)} \otimes W^{(1)} .
\end{aligned}
$$

Moreover,

$$
V^{(1)} \ni g \cdot \partial \otimes v=\partial \otimes(g \cdot v) \mapsto g \cdot v \in V .
$$

Finally, let $V=\operatorname{span}_{\mathbf{k}_{0}}\left\{v_{1}, \ldots, v_{n}\right\}, V^{*}=\operatorname{span}_{\mathbf{k}_{0}}\left\{v_{1}^{*}, \ldots, v_{n}^{*}\right\}, v=\sum a_{i} v_{i}$, and $g \cdot v_{j}^{*}=\sum g_{j i} v_{i}^{*}$. We then have

$$
v_{j}^{*}\left(g^{-1} v\right)=: v_{j}^{*}\left(\sum c_{k} v_{k}\right)=c_{j} .
$$

Hence,

$$
\begin{aligned}
c_{j}=v_{j}^{*}\left(g^{-1} v\right) & =\left(g \cdot v_{j}^{*}\right)(v)=\sum g_{j i} v_{i}^{*}(v)=\sum g_{j i} a_{i} . \\
\left(V^{*}\right)^{(1)} \ni g \cdot\left(\partial \otimes v_{j}^{*}\right) & =\partial \otimes\left(g \cdot v_{j}^{*}\right)=\partial \otimes \sum g_{j i} v_{i}^{*}= \\
& =\sum\left(g_{j i} \partial \otimes v_{i}^{*}+\partial\left(g_{j i}\right) \otimes v_{i}^{*}\right) \mapsto \\
& \mapsto \sum\left(g_{j i} F\left(v_{i}^{*}\right)+\partial\left(g_{j i}\right) \otimes\left(\partial \otimes v_{i}\right)^{*}\right) \in\left(V^{(1)}\right)^{*} .
\end{aligned}
$$

Now,

$$
\begin{aligned}
\sum\left(g_{j i} F\left(v_{i}^{*}\right)+\partial\left(g_{j i}\right) \otimes\left(\partial \otimes v_{i}\right)^{*}\right)(1 \otimes v) & =\sum g_{j i} a_{i}=c_{j}= \\
& =F\left(v_{j}^{*}\right)\left(g^{-1} \cdot(1 \otimes v)\right)= \\
& =\left(g \cdot F\left(v_{j}^{*}\right)\right)(1 \otimes v)
\end{aligned}
$$


and

$$
\begin{aligned}
\sum\left(g_{j i} F\left(v_{i}^{*}\right)+\partial\left(g_{j i}\right) \otimes\left(\partial \otimes v_{i}\right)^{*}\right)(\partial \otimes v) & =\sum g_{j i} \partial\left(a_{i}\right)+\partial\left(g_{j i}\right) a_{i}= \\
& =\partial\left(\sum g_{j i} a_{i}\right)= \\
& =\partial c_{j}=\partial\left(v_{j}^{*}\left(g^{-1} \cdot v\right)\right)= \\
& =F\left(v_{j}^{*}\right)\left(\partial \otimes\left(g^{-1} \cdot v\right)\right)= \\
& =\left(g \cdot F\left(v_{j}^{*}\right)\right)(\partial \otimes v) .
\end{aligned}
$$

In addition,

$$
\left(V^{*}\right)^{(1)} \ni g \cdot\left(1 \otimes v_{j}^{*}\right)=\sum g_{j i} \otimes v_{i}^{*} \mapsto \sum g_{j i}\left(\partial \otimes v_{i}\right)^{*}
$$

and

$$
\begin{aligned}
\sum g_{j i}\left(\partial \otimes v_{i}\right)^{*}(1 \otimes v) & =0=\sum\left(\partial \otimes v_{j}\right)^{*}\left(c_{i} \otimes v_{i}\right)=g \cdot\left(\partial \otimes v_{j}\right)^{*}(1 \otimes v), \\
\sum g_{j i}\left(\partial \otimes v_{i}\right)^{*}(\partial \otimes v) & =\sum g_{j i} a_{i}=c_{j}=\left(\partial \otimes v_{j}\right)^{*}\left(\partial \otimes\left(g^{-1} \cdot v\right)\right)= \\
& =g \cdot\left(\partial \otimes v_{j}\right)^{*}(\partial \otimes v) .
\end{aligned}
$$

This shows that all these maps are $G$-morphisms, which is all that we needed.

\subsection{Recovering a bialgebra over $\mathbf{k}_{\mathbf{0}}$.}

Remark 3. Let $\mathcal{C}=\boldsymbol{R e p}_{G}$ for a linear differential algebraic group $G$ and $\omega$ is the forgetful functor. This is one of main applications of the theory we are developing. It turns out that one can explicitly recover the group $G$ knowing $\mathcal{C}$. The paper [17] does this.

Let $X$ be an object of $\mathcal{C}$ and $\{\{X\}\}$ (respectively, $\mathcal{C}_{X}$ ) be the full abelian (respectively, full abelian tensor) subcategory of $\mathcal{C}$ generated by $X$ (respectively, containing $X$ and closed under $F$ ).

Denote by $B_{X}$ the object $P$ (or $P^{\prime}$ ) contained in $X^{*} \otimes \omega(X)$ and constructed as in $\left[9\right.$, Lemma 2.12] for the category $\{\{X\}\}$. Its image $\omega\left(B_{X}\right)$ stabilizes all $\omega(Y)$, where $Y$ is an object of $\{\{X\}\}$. Denote $P_{X}=\left(B_{X}\right)^{*}$. We note that $A_{X}:=\omega\left(P_{X}\right)$ is a finite dimensional vector space over $\mathbf{k}_{\mathbf{0}}$. One can construct $P_{X}$ directly as follows. Consider

$$
F_{X}=\bigoplus_{V \in \mathcal{O} b(\{\{X\}\})} V \otimes \omega(V)^{*} .
$$

For an object $V$ of $\{\{X\}\}$ we have the canonical injections:

$$
i_{V}: V \otimes \omega(V)^{*} \rightarrow F_{X} .
$$

Consider the minimal subobject $R_{X}$ of $F_{X}$ with subobjects

$$
\left\{\left(i_{V}\left(\mathrm{id} \otimes \phi^{*}\right)-i_{W}(\phi \otimes \mathrm{id})\right)\left(V \otimes \omega(W)^{*}\right) \mid V, W \in \mathcal{O b}(\{\{X\}\}), \phi \in \operatorname{Hom}(V, W)\right\} .
$$

We let

$$
P_{X}=F_{X} / R_{X}
$$

We now put

$$
A_{X}=\omega\left(P_{X}\right),
$$

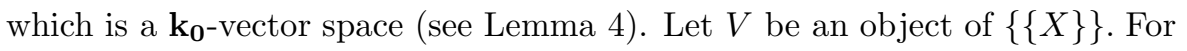

$$
v \in \omega(V), u \in \omega(V)^{*}
$$


we denote by

$$
a_{V}(v \otimes u)
$$

the image in $A_{X}$ of

$$
\omega\left(i_{V}\right)(v \otimes u) .
$$

So, for any $\phi \in \operatorname{Hom}(V, W)$ we have

$$
a_{V}\left(v \otimes \omega(\phi)^{*}(u)\right)=a_{W}(\omega(\phi)(v) \otimes u) .
$$

for all

$$
v \in \omega(V), u \in \omega(W)^{*} .
$$

Let us define a comultiplication on $A_{X}$. Let $\left\{v_{i}\right\}$ be a basis of $\omega(V)$ with the dual basis $\left\{u_{j}\right\}$ of $\omega(V)^{*}$. We let

$$
\Delta: a_{V}(v \otimes u) \mapsto \sum a_{V}\left(v_{i} \otimes u\right) \otimes a_{V}\left(v \otimes u_{i}\right) .
$$

The counit is defined in the following way:

$$
\varepsilon: a_{V}(v \otimes u) \mapsto u(v)
$$

The coinverse is defined as follows:

$$
S: a_{V}(v \otimes u) \mapsto a_{V^{*}}(u \otimes v) .
$$

Proposition 1. With the operations (11), (12), and (13) the finite dimensional $\mathbf{k}_{\mathbf{0}}$-vector space $A_{X}$ is a bialgebra.

Proof. This follows directly from [17, Lemmas 9, 10, and 11]. In these lemmas it is shown that the operations given by formulas (11), (12), and (13) are well defined and satisfy the usual axioms of a bialgebra. Moreover, in the proofs only the fact that the category is rigid and abelian was used.

\subsection{Differential structure.}

Definition 9. For a $\partial$-k $\mathbf{k}_{\mathbf{0}}$-algebra $R$ we define a group $\mathbf{A u t}^{\otimes, \partial}(\omega)(R)$ to be the set of sequences

$$
\lambda(R)=\left(\lambda_{X} \mid X \in \mathcal{O} \mathrm{b}(\mathcal{C})\right) \in \mathbf{A u t}^{\otimes, \partial}(\omega)(R)
$$

such that $\lambda_{X}$ is an $R$-linear automorphism of $\omega(X) \otimes R$ for each object $X, \omega(X) \in$ $\mathcal{O b}(\mathcal{V})$, that is, $\lambda_{X} \in \mathbf{A u t}_{R}(\omega(X) \otimes R)$, such that

- for all $X_{1}, X_{2}$ we have

$$
\lambda_{X_{1} \otimes X_{2}}=\lambda_{X_{1}} \otimes \lambda_{X_{2}}
$$

- $\lambda_{1}$ is the identity map on $\underline{1} \otimes R=R$,

- for every $\alpha \in \operatorname{Hom}(X, Y)$ we have

$$
\lambda_{Y} \circ\left(\alpha \otimes \operatorname{id}_{R}\right)=\left(\alpha \otimes \operatorname{id}_{R}\right) \circ \lambda_{X}: \omega(X) \otimes R \rightarrow \omega(Y) \otimes R,
$$

- for every $X$ we have

$$
\partial \circ \lambda_{X}=\lambda_{X^{(1)}} \circ \partial
$$

- the group operation $\lambda_{1}(R) \cdot \lambda_{2}(R)$ is defined by composition in each set $\operatorname{Aut}_{R}(\omega(X) \otimes R)$. 
Example 6. For $\mathcal{C}=\operatorname{Rep}_{G}$, where $G$ is a linear differential algebraic group, we will construct an injection $G \rightarrow \mathbf{A u t}^{\otimes, \partial}(\omega)$. For this take any differential $\mathbf{k}_{\mathbf{0}^{-}}$-algebra $R$ and $g \in G(R)$. For any $V \in \mathbf{R e p}_{G}$ the element $g$ defines an $R$-linear automorphism

$$
\lambda_{V}: \omega(V) \otimes R \rightarrow \omega(V) \otimes R .
$$

We show that $\left\{\lambda_{V}\right\}$ is an element of $\mathbf{A u t}^{\otimes, \partial}(\omega)(R)$. Indeed, formulas (14), (15), and injectivity are the usual properties of representation. We show that formula (16) holds true as well:

$$
\begin{aligned}
\lambda_{X^{(1)}} \circ \partial(v \otimes r)= & \lambda_{X^{(1)}}((\partial \otimes v) \otimes r+(1 \otimes v) \otimes \partial r)=(\partial \otimes(g \cdot v)) \otimes r+ \\
& +(1 \otimes(g \cdot v)) \otimes \partial r)=\partial((g \cdot v) \otimes r)=\partial \circ \lambda_{X}(v \otimes r)
\end{aligned}
$$

for any $r \in R$. The less obvious part is surjectivity and instead of showing it directly we prove the main result using the dual language of the corresponding differential Hopf algebras.

Speaking informally, in the previous sections things looked quite algebraic without differential algebra. The differential part appears here: we have an additional condition (16), which allows one to prove the differential analogue of Tannaka's theorem. We are now going to look at the "differential" category $\mathcal{C}_{X}$ generated by $X$. For $X \in \mathcal{O b}(\mathcal{C})$ we let

$$
\mathcal{P}_{X}=\underset{Y \in \underset{\mathcal{O} \mathrm{b}\left(\mathcal{C}_{X}\right)}{\lim _{Y}}}{ } P_{Y}
$$

Let

We finally put

$$
\mathcal{B}_{X}=\omega\left(\mathcal{P}_{X}\right)
$$

$$
A=\lim _{X \in \overrightarrow{\mathcal{O b}}(\mathcal{C})} \mathcal{B}_{X} .
$$

This is a coalgebra due to the previous considerations. We are going to make it a differential Hopf algebra using the prolongation functor $F$ and $\otimes$.

For this take $v \in \omega(V), w \in \omega(W), u \in \omega(V)^{*}, t \in \omega(W)^{*}$ and let

$$
\left.a_{V}(v \otimes u) \cdot a_{W}(w \otimes t)=a_{V \otimes W}((v \otimes w)) \otimes(u \otimes t)\right) .
$$

Define

$$
F(u)(1 \otimes v):=u(v), F(u)(\partial \otimes v):=\partial(u(v)), u \in V^{*}, v \in V
$$

for a vector space $V$. Introduce a differential structure on $A$ :

$$
\partial\left(a_{V}(v \otimes u)\right)=a_{V^{(1)}}((\partial v) \otimes F(u)),
$$

which can be extended to the whole $A$ as a derivation. Recall that we extend here $u \in V^{*}$ to an element of $\left(V^{(1)}\right)^{*}$ by

$$
F(u)(\partial \otimes w):=\partial(u(w)), \quad F(u)(1 \otimes w):=u(w) .
$$

Note also that

$$
a_{V}(v \otimes u)=a_{V^{(1)}}\left(\partial v \otimes \varphi^{*}(u)\right) .
$$

We recall here that $\varphi^{*}$ is the dual map to the morphism given in (3):

$$
\phi: V^{(1)} \rightarrow V, \quad \varphi:=\omega(\phi): 1 \otimes v \mapsto 0, \partial \otimes v \mapsto v .
$$

The following "computational" version of (6) and (7) will further be used in Proposition 2. 
Lemma 6. Let $V \in \mathcal{O b}\left(\mathcal{C}_{X}\right)$ and $\left\{v_{1}, \ldots, v_{n}\right\}$ be an ordered basis of $\omega(V)$ corresponding to the splitting in (7) with the dual basis $\left\{v_{1}^{*}, \ldots, v_{n}^{*}\right\}$ of the dual vector space $\omega(V)^{*}$. Then,

$$
a_{\left(V^{*}\right)^{(1)}}\left(\partial\left(v_{j}^{*}\right) \otimes F\left(v_{i}\right)\right)=a_{\left(V^{(1)}\right)^{*}}\left(F\left(v_{j}^{*}\right) \otimes \partial v_{i}\right) .
$$

Proof. Let $g_{X}$ be the morphism $\left(V^{*}\right)^{(1)} \rightarrow V^{(1)^{*}}$ given by (6). We then have

$$
i_{\omega(V)^{*}}\left(v_{i}^{*}\right)=1 \otimes v_{i}^{*}
$$

and

$$
\varphi_{\omega(V)}{ }^{*}\left(v_{i}^{*}\right)\left(1 \otimes v_{j}\right)=0, \quad \varphi_{\omega(V)}{ }^{*}\left(v_{i}^{*}\right)\left(\partial \otimes v_{j}\right)=\delta_{i j} .
$$

Therefore,

$$
\varphi_{\omega(V)}{ }^{*}\left(v_{i}^{*}\right)=\left(\partial \otimes v_{i}\right)^{*}
$$

and

$$
\omega\left(g_{V}\right)\left(1 \otimes v_{i}^{*}\right)=\varphi_{\omega(V)}{ }^{*}\left(v_{i}^{*}\right)=\left(\partial \otimes v_{i}\right)^{*} .
$$

Now,

$$
\varphi_{\omega(V)^{*}}\left(\partial \otimes v_{i}^{*}\right)=v_{i}^{*}
$$

and for any $a_{1}, \ldots, a_{n} \in \mathbf{k}_{\mathbf{0}}$ we have

$$
i_{\omega(V)}^{*}:\left(1 \otimes v_{i}\right)^{*}+\sum_{j=1}^{n} a_{j} \cdot\left(\partial \otimes v_{j}\right)^{*} \mapsto v_{i}^{*} .
$$

Hence, there exist $b_{i 1}, \ldots, b_{i n} \in \mathbf{k}_{\mathbf{0}}$ such that

$$
\omega\left(g_{V}\right)\left(\partial \otimes v_{i}^{*}\right)=\left(1 \otimes v_{i}\right)^{*}+\sum_{j=1}^{n} b_{i j} \cdot\left(\partial \otimes v_{j}\right)^{*} .
$$

Since $\omega\left(g_{V}\right)$ respects the splittings into direct sums given in $(7), b_{i j}=0$ for all $1 \leqslant i, j \leqslant n$. Note that

$$
F\left(v_{i}^{*}\right)\left(1 \otimes v_{j}\right)=\delta_{i j}, \quad F\left(v_{i}^{*}\right)\left(\partial \otimes v_{j}\right)=\partial\left(v_{i}^{*}\left(v_{j}\right)\right)=0 .
$$

Therefore,

$$
F\left(v_{i}^{*}\right)=\left(1 \otimes v_{i}\right)^{*}=\omega\left(g_{V}\right)\left(\partial \otimes v_{i}^{*}\right) .
$$

Moreover,

$$
\omega\left(g_{V}\right)^{*}\left(\partial v_{i}\right)\left(1 \otimes v_{j}^{*}\right)=\left(\partial v_{i}\right)\left(\omega\left(g_{V}\right)\left(1 \otimes v_{j}^{*}\right)\right)=\partial v_{i}\left(\left(\partial v_{j}\right)^{*}\right)=\delta_{i j}
$$

and

$$
\omega\left(g_{V}\right)^{*}\left(\partial v_{i}\right)\left(\partial \otimes v_{j}^{*}\right)=\left(\partial v_{i}\right)\left(\omega\left(g_{V}\right)\left(\partial \otimes v_{j}^{*}\right)\right)=\left(1 \otimes v_{j}\right)^{*}\left(\partial v_{i}\right)=0 .
$$

Hence,

$$
\omega\left(g_{V}\right)^{*}\left(\partial v_{i}\right)=\left(1 \otimes v_{i}^{*}\right)^{*}
$$

Now,

Therefore,

$$
F\left(v_{i}\right)\left(1 \otimes v_{j}^{*}\right)=\delta_{i j}, \quad F\left(v_{i}\right)\left(\partial \otimes v_{j}^{*}\right)=0 .
$$

$$
F\left(v_{i}\right)=\left(1 \otimes v_{i}^{*}\right)^{*}=\omega\left(g_{V}\right)^{*}\left(\partial v_{i}\right),
$$

which is what we finally needed to apply (10).

Proposition 2. With the operations (17), (18), (11), (12), and (13) the $\mathbf{k}_{\mathbf{0}}$-vector space $A$ is a direct limit of finitely generated commutative associative differential Hopf algebras with the unity. 
Proof. All the statements follow from Proposition 1 and [17, Lemmas 5, 6, 7, 9, 10, and 11] except for finitely generated, because our construction of $\mathcal{B}_{X}$ and operations on it are the same as in [17]. The $\partial$ - $\mathbf{k}_{\mathbf{0}}$-algebra $\mathcal{B}_{X}$ is generated by the elements $a_{X}(v \otimes u)$. The statement now follows as $\omega(X) \otimes \omega(X)^{*}$ is a finite dimensional $\mathbf{k}_{\mathbf{0}}$-vector space.

In order to show the other properties like:

- $\partial, m, \Delta, \varepsilon, S$ are well defined;

- commutation with $\partial$ of $\Delta, S, \varepsilon$;

- respecting multiplication $(\Delta, S, \varepsilon$ are algebra homomorphisms);

- the product rule for the multiplication,

in these lemmas only the properties of a neutral differential Tannakian category were used. In particular, properties (4), (5), (6), and (7) were needed for the proofs. That is why one finds them among the axioms of a neutral differential Tannakian category. Moreover, we have Lemma 6, which is used in [17, Lemma 11] to show that the coinverse we have defined commutes with differentiation $\partial$.

\subsection{Equivalence of categories.}

Lemma 7. The restriction $\left.\omega\right|_{\mathcal{C}_{X}}: \mathcal{C}_{X} \rightarrow \mathcal{V}$ factors through $\mathbf{C o D i f f}_{\mathcal{B}_{X}}$.

Proof. Let $Y$ be an object of $\mathcal{C}_{X}$. We introduce a $\mathcal{B}_{X}$-comodule structure on $\omega(Y)$.

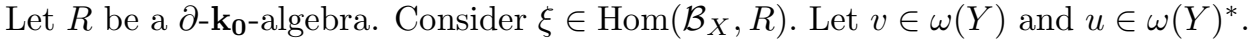
There is an endomorphism $\lambda_{Y}$ of $\omega(Y) \otimes R$ such that

$$
\left\langle\lambda_{Y}(v), u\right\rangle=u\left(\lambda_{Y}(v)\right)=\xi\left(a_{Y}(v \otimes u)\right) .
$$

Put $R=\mathcal{B}_{X}$ and $\xi=\operatorname{id}_{\mathcal{B}_{X}}$. We obtain a $\mathcal{B}_{X}$-linear map

$$
\lambda_{Y}: \omega(Y) \otimes \mathcal{B}_{X} \rightarrow \omega(Y) \otimes \mathcal{B}_{X} .
$$

Composing $\lambda_{Y}$ with the imbedding

$$
\omega(Y) \rightarrow \omega(Y) \otimes \mathcal{B}_{X}, \quad v \mapsto v \otimes a_{\underline{1}}(e \otimes f),
$$

where $\{e\}$ is a basis of $\omega(\underline{1})$ and $f(e)=1$, we provide a $\mathcal{B}_{X}$-comodule structure

$$
\rho_{Y}: \omega(Y) \rightarrow \omega(Y) \otimes \mathcal{B}_{X}
$$

on $\omega(Y)$. Moreover, if $Y_{1}, Y_{2} \in \mathcal{O b}\left(\mathcal{C}_{X}\right)$ and $\varphi \in \operatorname{Hom}\left(Y_{1}, Y_{2}\right)$ then we have

$$
\begin{aligned}
\left\langle\lambda_{Y_{2}} \circ \omega(\varphi)(v), u\right\rangle & =\xi\left(a_{Y_{2}}(\omega(\varphi)(v) \otimes u)\right)=\xi\left(a_{Y_{1}}\left(v \otimes \omega(\varphi)^{*}(u)\right)\right)= \\
& =\left\langle\lambda_{Y_{1}}(v), \omega(\varphi)^{*}(u)\right\rangle=\left\langle\omega(\varphi) \circ \lambda_{Y_{1}}(v), u\right\rangle
\end{aligned}
$$

for all $v \in \omega\left(Y_{1}\right)$ and $u \in \omega\left(Y_{2}\right)^{*}$. Hence,

$$
\lambda_{Y_{2}} \circ \omega(\varphi)=\omega(\varphi) \circ \lambda_{Y_{1}} .
$$

Therefore, the diagram

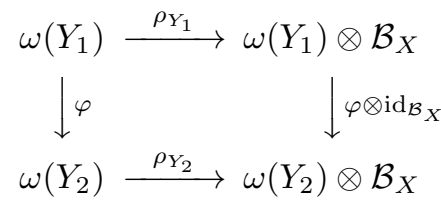

is commutative. This implies that we have defined a functor $\mathcal{C}_{X} \rightarrow \operatorname{CoDiff} \mathcal{B}_{X}$. The forgetful functor $\operatorname{CoDiff}_{\mathcal{B}_{X}} \rightarrow \mathcal{V}$ closes the commutative diagram.

Proposition 3. Let $\omega: \mathcal{C} \rightarrow \mathcal{V}$. Then $\omega$ defines an equivalence of categories $\mathcal{C}_{X} \rightarrow \operatorname{CoDiff}_{\mathcal{B}_{X}}$ taking $\left.\omega\right|_{\mathcal{C}_{X}}$ to the forgetful functor. 
Proof. For an object $Y$ of $\mathcal{C}_{X}$ it is shown in Lemma 7 how to put a $\mathcal{B}_{X}$-comodule structure on $\omega(Y)$. We first demonstrate that the induced functor

$$
\left.\omega\right|_{\mathcal{C}_{X}}: \mathcal{C}_{X} \rightarrow \operatorname{CoDiff}_{\mathcal{B}_{X}}
$$

is essentially surjective. For a differential comodule $V$ over $\mathcal{B}_{X}$ consider the object $\mathcal{P}_{X} \otimes V$. It has a subobject $S_{X}$ generated by the kernel of the morphism

$$
(\varepsilon \circ \omega) \otimes \operatorname{id}_{V}: \mathcal{P}_{X} \otimes V \rightarrow \mathbf{k}_{\mathbf{0}} \otimes V \cong V .
$$

We then have

$$
\omega\left(\left(\mathcal{P}_{X} \otimes V\right) / S_{X}\right)=\left(\omega\left(\mathcal{P}_{X}\right) \otimes V\right) / \omega\left(S_{X}\right)=\left(\mathcal{B}_{X} \otimes V\right) / \omega\left(S_{X}\right) \cong V .
$$

Denote $\mathcal{P}_{X} \otimes V / S_{X}=V_{X}$. Now, $V_{X}$ consists of the collection $\left(Y_{\alpha}\right)$ of objects $Y_{\alpha}$ in $\mathcal{C}_{X}$ with isomorphisms $\phi_{\beta, \alpha}: Y_{\alpha} \rightarrow Y_{\beta}$. For any $\alpha$ we then have $\omega\left(Y_{\alpha}\right) \cong V$. Hence, $\left.\omega\right|_{\mathcal{C}_{X}}$ is essentially surjective.

We show now that $\left.\omega\right|_{\mathcal{C}_{X}}$ is full. For $X_{1}, X_{2} \in \mathcal{O b}\left(\mathcal{C}_{X}\right)$ consider the comodules $V_{1}$ and $V_{2}$ corresponding to $X_{1}$ and $X_{2}$, respectively. Let $\varphi \in \operatorname{Hom}\left(V_{1}, V_{2}\right)$. There is a map (see Lemma 7)

$$
\psi_{\varphi}:=\mathrm{id} \otimes \varphi: \mathcal{P}_{X} \otimes V_{1} \rightarrow \mathcal{P}_{X} \otimes V_{2},
$$

which is a morphism due to Lemmas 2 and 3 with $V=V_{1}, T=\mathcal{P}_{X} \otimes V_{2}$, and $X=\mathcal{P}_{X}$. This induces a morphism

$$
\left(V_{1}\right)_{X} \rightarrow\left(V_{2}\right)_{X}
$$

By the definition of $\mathcal{S}_{X}$ we have surjections

$$
\operatorname{id} \otimes \text { ev }: \mathcal{P}_{X} \otimes \omega\left(X_{i}\right) \rightarrow X_{i}
$$

that induce isomorphisms

$$
\left(V_{i}\right)_{X} \rightarrow X_{i}
$$

Again, $\mathcal{P}_{X} \otimes V_{i}$ are the collections $\left(Y_{\alpha}^{i}\right)$ and, hence, this $\psi$ induces a map $Y_{\alpha}^{1} \rightarrow Y_{\alpha}^{2}$. So, we have a morphism $X_{1} \rightarrow X_{2}$ corresponding to $\varphi$.

Since $\omega$ is faithful, we have an equivalence of categories $\mathcal{C}_{X} \rightarrow \operatorname{Comod}_{\mathcal{B}_{X}}$. Finally, the functor $\mathcal{C}_{X} \rightarrow \operatorname{CoDiff}_{\mathcal{B}_{X}}$ respects the functor $F$, because $\omega$ commutes with $F$ by definition. This establishes the required equivalence of categories.

Proposition 4. For each object $X$ of the category $\mathcal{C}$ the identification of $\mathcal{C}_{X}$ with CoDiff $_{\mathcal{B}_{X}}$ provides an equivalence of categories $\mathcal{C} \rightarrow \operatorname{CoDiff}_{A}$ taking $\omega$ to the forgetful functor from $\mathbf{C o D i f f}_{A}$ to $\mathcal{V}$.

Proof. Follows from Proposition 3. Indeed, for subcategories $\mathcal{C}_{1} \subset \mathcal{C}_{2} \subset \mathcal{C}$ there is a restriction map $\varphi_{1,2}:$ End $\left(\left.\omega\right|_{\mathcal{C}_{2}}\right) \rightarrow \underline{\text { End }}\left(\left.\omega\right|_{\mathcal{C}_{1}}\right)$. Hence, we have the induced $\operatorname{map} \varphi_{1,2}{ }^{*}:\left(\underline{\text { End }}\left(\left.\omega\right|_{\mathcal{C}_{1}}\right)\right)^{*} \rightarrow\left(\underline{\text { End }}\left(\left.\omega\right|_{\mathcal{C}_{2}}\right)\right)^{*}$. Moreover, $(\mathcal{C}, \omega)=\underline{\lim }\left(\mathcal{C}_{X},\left.\omega\right|_{\mathcal{C}_{X}}\right)$ with respect to the injective system of inclusion maps.

Corollary 1. The group $G_{X}$, defined by $G_{X}(R)=\operatorname{Hom}\left(\mathcal{B}_{X}, R\right)$ for each $\partial-\mathbf{k}_{\mathbf{0}}$ algebra $R$, is a linear differential algebraic group.

Proof. By Proposition 2 the differential Hopf algebra $\mathcal{B}_{X}$ is finitely generated in the differential sense. Thus, according to the proof of [2, Proposition 12, page 914] it is a coordinate ring of a linear differential algebraic group.

Corollary 2. For the linear differential algebraic group $G_{X}$ defined above we have

$$
\operatorname{Aut}^{\otimes, \partial}\left(\left.\omega\right|_{\mathcal{C}_{X}}\right) \cong G_{X} .
$$


Proof. From Proposition 3 we have equivalence of categories

$$
\mathcal{C}_{X} \cong \operatorname{CoDiff}_{\mathcal{B}_{X}}
$$

with $\left.\omega\right|_{\mathcal{C}_{X}}$ corresponding to the forgetful functor $\omega^{\prime}$ from $\operatorname{CoDiff}_{\mathcal{B}_{X}}$ to $\mathcal{V}$. Moreover, [17, Theorem 2] says that the linear differential algebraic group

$$
G_{X} \cong \mathbf{A u t}^{\otimes, \partial}\left(\omega^{\prime}\right)
$$

which concludes the proof, as $\mathbf{A u t}^{\otimes, \partial}\left(\omega^{\prime}\right) \cong \mathbf{A u t}^{\otimes, \partial}\left(\left.\omega\right|_{\mathcal{C}_{X}}\right)$.

\section{MAIN THEOREM}

Theorem 2. Let $(\mathcal{C}, \omega)$ be a neutral differential Tannakian category. Then

$$
(\mathcal{C}, \omega) \cong \operatorname{Rep}_{G}
$$

for the differential group scheme

$$
G=\mathbf{A u t}^{\otimes, \partial}(\omega)
$$

which is

(1) represented by the commutative associative differential Hopf algebra $A=$ $\lim _{\longrightarrow} \mathcal{B}_{X}$

(2) a pro-linear differential algebraic group.

Proof. The first statement is contained in Proposition 4. Property (1) follows from Corollary 2 by taking limits. According to Corollary 1 for each object $X$ of $\mathcal{C}$ the group $G_{X}$ is a linear differential algebraic group. Property (2) now follows.

\section{Applications}

We will show how one can apply the theory developed above to parametric linear differential equations. The theory of parameterized linear differential equations considers equations of the form

$$
\frac{\partial Y}{\partial x}=A(t, x) Y
$$

where $A$ is an $n \times n$ matrix whose entries are functions of $x$ and of a parameter $t$ and was developed in detail in [4]. Formally one considers a differential field $\mathbf{k}$ of characteristic zero with commuting derivations $\Delta=\left\{\partial_{t}, \partial_{x}\right\}$ and we assume that $\mathbf{k}_{\mathbf{0}}=\left\{c \in \mathbf{k} \mid \partial_{x}(c)=0\right\}$, the constants with respect to $\partial_{x}$, forms a differentially closed $\partial_{t}$-field ${ }^{3}$. Given a linear differential equation $\partial_{x} Y=A Y$, where $A$ is an $n \times n$ matrix with entries in $\mathbf{k}$, there exists a $\Delta$-differential field extension $K$ of $\mathbf{k}$ having the same $\partial_{x}$-constants as $\mathbf{k}$ and where $K$ is generated (as a $\Delta$-differential field) over $\mathbf{k}$ by the entries of an $n \times n$ invertible matrix $Z$ satisfying $\partial_{x} Z=A Z$ (Theorem 3.5, [4]). This field is called the parameterized Picard-Vessiot extension associated to the equation and is unique up to differential $\mathbf{k}$-isomorphism. The group of $\mathbf{k}$-automorphisms of $K$ commuting with the derivations in $\Delta$ is called the parameterized Picard-Vessiot group of the equation and can be shown to be a linear differential algebraic group. The goal of this section is to define this group using the theory developed in the previous sections.

\footnotetext{
${ }^{3}$ In [4], larger sets of derivations are considered but, for simplicity we shall consider one parametric derivation and one principal derivation. The subscripts $t$ and $x$ are a convenient way of distinguishing these but we do not assume that we are dealing with functions of variables $t$ and $x$.
} 
5.1. Preliminaries. Let $\mathbf{k}, \Delta, \mathbf{k}_{\mathbf{0}}$ be as above with $\mathbf{k}_{\mathbf{0}}$ differentially closed. The field of constants of $\mathbf{k}_{\mathbf{0}}$ w.r.t. the differential operator $\partial_{t}$ is denoted by $\mathbf{k}_{\mathbf{0}}{ }^{\left\{\partial_{t}\right\}}$. A $\partial_{x}$-k-module $M$ is a finite dimensional $\mathbf{k}$-vector space with a linear operator $\partial_{x}$ satisfying the product rule:

$$
\partial_{x}(r m)=\left(\partial_{x} r\right) m+r \partial_{x} m
$$

for all $r \in \mathbf{k}$ and $m \in M$. Starting from Section 5.2 we will also use the notation $M^{(0)}$ for such a module $M$.

Let $\left\{e_{1}, \ldots, e_{n}\right\}$ be a $\mathbf{k}$-basis of $M$. Define the elements $a_{i j} \in \mathbf{k}$ by

$$
\partial_{x} e_{i}=-\sum_{j=1}^{n} a_{j i} e_{j}
$$

where $1 \leqslant i \leqslant n$. Let $u=a_{1} e_{1}+\ldots+a_{n} e_{n}$. Then $\partial_{x} u=0$ iff

$$
\sum_{i=1}^{n} \partial_{x}\left(a_{i}\right) e_{i}-\sum_{i=1}^{n} \sum_{j=1}^{n} a_{j} a_{i j} e_{i}=0
$$

or, equivalently,

$$
\partial_{x}\left(\begin{array}{c}
a_{1} \\
\vdots \\
a_{n}
\end{array}\right)=A\left(\begin{array}{c}
a_{1} \\
\vdots \\
a_{n}
\end{array}\right),
$$

where $A=\left(\begin{array}{ccc}a_{11} & \ldots & a_{1 n} \\ \vdots & \ddots & \vdots \\ a_{n 1} & \ldots & a_{n n}\end{array}\right)$.

We are going to define a formal object associated with a parametric linear differential equation, e.g.,

$$
\partial_{x} y=\frac{t}{x} y
$$

which is going to be equivalent to the equation in some sense and will allow us to recover the parametric differential Galois group $G$ from getting first the category of all finite dimensional differential rational representations of $G$ and then taking all coordinate functions of representations. We obtain the algebra $A:=\mathbf{k}_{0}\{G\}$ of differential algebraic functions on $G$, then recover derivation $\partial$ on $A$, comultiplication $\Delta: A \rightarrow A \otimes A$, e.t.c.

Remark 4. In what follows we will be using higher order derivation functors (of order 2 and higher $)^{4}$. Formally, this is not the same as to iterate the first order derivation functor, but:

- it produces objects of smaller dimension than the iterated derivatives (the derivation functor $F$ applied several times) and

- captures the same differential information as we shall see in Proposition 7.

Although it is not necessary to use higher order derivation functors, we hope that this Tannakian approach will eventually produce algorithms computing Galois groups of systems of linear differential equations with parameters and smaller objects are more desirable for computation.

\footnotetext{
${ }^{4}$ see Definition 1.
} 
The construction where $M^{(i)}$ is the $\partial_{x}$-module with the matrix obtained by the $i$ th prolongation (parallel to the differential-difference construction of $[5,6,7]$ )

$$
A_{i}=\left(\begin{array}{ccccc}
A & 0 & 0 & \ldots & 0 \\
A_{t} & A & 0 & \ldots & 0 \\
A_{t t} & 2 A_{t} & A & \ldots & 0 \\
\vdots & \vdots & \vdots & \ddots & \vdots \\
A_{t^{i}} & \left(\begin{array}{l}
i \\
1
\end{array}\right) A_{t^{i-1}} & \left(\begin{array}{l}
i \\
2
\end{array}\right) A_{t^{i-2}} & \ldots & A
\end{array}\right)
$$

of $A_{0}=A=\left(\begin{array}{ccc}a_{11} & \ldots & a_{1 n} \\ \vdots & \ddots & \vdots \\ a_{n 1} & \ldots & a_{n n}\end{array}\right)$ does look like an adequate one. In the next section we will see how to treat all these $M^{(i)}, i \geqslant 0$. It turns out that we can proceed in two ways:

(1) compute the $i$ th prolongation $A_{i}$ of the matrix $A$,

(2) compute the $i$ th prolongation $M^{(i)}$ of the $\partial_{x}$-k-module $M$.

Both approaches give the same $\partial_{x}$-structure as Proposition 5 shows.

5.2. The category $\mathcal{M}$. We first give a coordinate free definition and then introduce a canonical basis and use this constructive approach to develop our theory.

Definition 10. We let

(1) an object $M$ of $\mathcal{M}$ be an object of $\mathcal{V}$ together with a differential module structure given by $\partial_{x}$ which commutes with $\partial$,

(2) morphisms between objects of $\mathcal{M}$ be those which commute with the action of $\partial_{x}:$

$$
\operatorname{Hom}(M, N)=\operatorname{Hom}_{\mathbf{k}\left[\partial_{x}\right]}(M, N)
$$

for all objects $M$ and $N$ of $\mathcal{M}$,

(3) subobjects, $\otimes, \oplus$, and ${ }^{*}$ are as in the category of differential modules $[15$, Section 2.2]

(4) the functors $F^{p}$ are the same as in $\mathcal{V}$.

Remark 5. Recall that

$$
M^{(i)}=\mathbf{k}[\partial]_{\leqslant i} \otimes M^{(0)},
$$

where $M^{(0)}$ is another notation for $M$.

5.3. The equations-modules correspondence. Derivation $\partial_{x}$ has to be defined on the prolongations of $M$ in such a way that is commutes with the map $\partial: M \rightarrow$ $M^{(1)}, v \mapsto \partial \otimes v$.

Lemma 8. For an object $M$, with the $\partial_{x}$-structure given by an $n \times n$ matrix $A$ with respect to an ordered basis $\left\{e_{1}, \ldots, e_{n}\right\}$, the matrix $A_{i+1}$ corresponding to the action of $\partial_{x}$ on $M^{(i+1)}$ with respect to the ordered basis

$$
\left\{\partial^{i+1} e_{1}, \ldots, \partial^{i+1} e_{n}, \ldots, e_{1}, \ldots, e_{n}\right\}
$$


is of the form

$$
\tilde{A}_{i+1}=\left(\begin{array}{ccccc}
A & 0 & 0 & \ldots & 0 \\
\left(\begin{array}{c}
i+1 \\
1
\end{array}\right) A_{t} & A & 0 & \ldots & 0 \\
\left(\begin{array}{c}
++1 \\
2
\end{array}\right) A_{t t} & \left(\begin{array}{l}
i \\
1
\end{array}\right) A_{t} & A & \ldots & 0 \\
\vdots & \vdots & \vdots & \ddots & \vdots \\
A_{t^{i+1}} & \left(\begin{array}{c}
i \\
i
\end{array}\right) A_{t^{i}} & A_{t^{i-1}} & \ldots & A
\end{array}\right)
$$

Proof. Let

$$
A_{0}=A=\left(\begin{array}{ccc}
a_{11} & \ldots & a_{1 n} \\
\vdots & \ddots & \vdots \\
a_{n 1} & \ldots & a_{n n}
\end{array}\right)
$$

be the matrix for $\partial_{x}$ in the module $M^{(0)}$. For $i=1$ the basis of the module $M^{(1)}$ is $\left\{\partial e_{1}, \ldots, \partial e_{n}, e_{1}, \ldots, e_{n}\right\}$. For $k, 1 \leqslant k \leqslant n$, we have

$$
\begin{aligned}
\partial_{x}\left(\partial e_{k}\right)=\partial\left(\partial_{x}\left(e_{k}\right)\right)=\partial\left(-\sum_{p=1}^{n} a_{p k} e_{p}\right) & =-\sum_{p=1}^{n} a_{p k} \partial e_{p}-\sum_{p=1}^{n}\left(\partial_{t} a_{p k}\right) e_{p}, \\
\partial_{x}\left(e_{k}\right) & =-\sum_{p=1}^{n} a_{p k} e_{p} .
\end{aligned}
$$

Assume the result for $i=m$. We have $\left\{\partial^{m+1} e_{1}, \ldots, \partial^{m+1} e_{n}, \ldots, e_{1}, \ldots, e_{n}\right\}$ as the ordered basis of $M^{(m+1)}$. For each $l, 1 \leqslant l \leqslant n$, we have

$$
\begin{aligned}
\partial_{x} & \left(\partial^{m+1} e_{l}\right)=\partial\left(\partial_{x}\left(\partial^{m} e_{l}\right)\right)= \\
& =\partial\left(-\sum_{q=0}^{m} \sum_{r=1}^{n}\left(\begin{array}{c}
m \\
q
\end{array}\right)\left(\partial_{t}^{q} a_{r l}\right) \partial^{m-q} e_{r}\right)= \\
& =-\sum_{q=0}^{m} \sum_{r=1}^{n}\left(\begin{array}{c}
m \\
q
\end{array}\right)\left(\partial_{t}^{q+1} a_{r l}\right) \partial^{m-q} e_{r}-\sum_{q=0}^{m} \sum_{r=1}^{n}\left(\begin{array}{c}
m \\
q
\end{array}\right)\left(\partial_{t}^{q} a_{r l}\right) \partial\left(\partial^{m-q} e_{r}\right)= \\
& =-\sum_{q=0}^{m} \sum_{r=1}^{n}\left(\begin{array}{c}
m \\
q
\end{array}\right)\left(\partial_{t}^{q+1} a_{r l}\right) \partial^{m+1-(q+1)} e_{r}-\sum_{q=0}^{m} \sum_{r=1}^{n}\left(\begin{array}{c}
m \\
q
\end{array}\right)\left(\partial_{t}^{q} a_{r l}\right) \partial^{m+1-q} e_{r}= \\
& =-\sum_{q=1}^{m+1} \sum_{r=1}^{n}\left(\begin{array}{c}
m \\
q-1
\end{array}\right)\left(\partial_{t}^{q} a_{r l}\right) \partial^{m+1-q} e_{r}-\sum_{q=0}^{m} \sum_{r=1}^{n}\left(\begin{array}{c}
m \\
q
\end{array}\right)\left(\partial_{t}^{q} a_{r l}\right) \partial^{m+1-q} e_{r}= \\
& =-\sum_{q=0}^{m+1} \sum_{r=1}^{n}\left(\begin{array}{c}
m+1 \\
q
\end{array}\right)\left(\partial_{t}^{q} a_{r l}\right) \partial^{m+1-q} e_{r},
\end{aligned}
$$

because $\left(\begin{array}{c}m \\ q-1\end{array}\right)+\left(\begin{array}{c}m \\ q\end{array}\right)=\left(\begin{array}{c}m+1 \\ q\end{array}\right)$.

Proposition 5. For an object $M$ of $\mathcal{M}$ there exists an ordered basis of each $M^{(i)}$ such that the matrix $A_{i}$ corresponding to the action of $\partial_{x}$ w.r.t. this basis is of the 
form

$$
A_{i}=\left(\begin{array}{ccccc}
A & 0 & 0 & \ldots & 0 \\
A_{t} & A & 0 & \ldots & 0 \\
A_{t t} & \left(\begin{array}{l}
2 \\
1
\end{array}\right) \cdot A_{t} & A & \ldots & 0 \\
\vdots & \vdots & \vdots & \ddots & \vdots \\
A_{t^{i}} & \left(\begin{array}{l}
i \\
1
\end{array}\right) A_{t^{i-1}} & \left(\begin{array}{l}
i \\
2
\end{array}\right) A_{t^{i-2}} & \ldots & A
\end{array}\right)
$$

where

$$
A_{0}=A=\left(\begin{array}{ccc}
a_{11} & \ldots & a_{1 n} \\
\vdots & \ddots & \vdots \\
a_{n 1} & \ldots & a_{n n}
\end{array}\right)
$$

is the matrix for $\partial_{x}$ in the module $M$.

Proof. According to Lemma 8 for each $M^{(i)}$ there exists an ordered basis

$$
\left\{\partial^{i} e_{1}, \ldots, \partial^{i} e_{n}, \ldots, e_{1}, \ldots, e_{n}\right\}
$$

such that the matrix corresponding to $\partial_{x}$ is

$$
\tilde{A}_{i}=\left(\begin{array}{ccccc}
A & 0 & 0 & \ldots & 0 \\
\left(\begin{array}{c}
i \\
1
\end{array}\right) \cdot A_{t} & A & 0 & \ldots & 0 \\
\left(\begin{array}{c}
l \\
2
\end{array}\right) \cdot A_{t t} & \left(\begin{array}{c}
i-1 \\
1
\end{array}\right) \cdot A_{t} & A & \ldots & 0 \\
\vdots & \vdots & \vdots & \ddots & \vdots \\
A_{t^{i}} & \ldots & \ldots & \ldots & A
\end{array}\right)
$$

Denote this basis by $\left\{f_{1}, \ldots, f_{(i+1) \cdot n}\right\}$. Note that if we change a basis:

$$
\left(\begin{array}{c}
g_{1} \\
\vdots \\
g_{(i+1) \cdot n}
\end{array}\right)=C \cdot\left(\begin{array}{c}
f_{1} \\
\vdots \\
f_{(i+1) \cdot n}
\end{array}\right)
$$

and all entries of the matrix $C$ are constants then the corresponding matrix $A_{i}$ must be replaced by $\left(C^{T}\right)^{-1} A_{i} C^{T}$. Consider the basis $\left\{g_{1}, \ldots, g_{(i+1) \cdot n}\right\}$ given by the following change-of-basis matrix

$$
C:=\left(\begin{array}{ccccc}
E & 0 & 0 & \ldots & 0 \\
E & \left(\begin{array}{c}
i \\
1
\end{array}\right) \cdot E & 0 & \ldots & 0 \\
E & \left(\begin{array}{c}
i-1 \\
1
\end{array}\right) \cdot E & \left(\begin{array}{l}
i \\
2
\end{array}\right) \cdot E & \ldots & 0 \\
\vdots & \vdots & \vdots & \ddots & \vdots \\
E & 2 \cdot E & 3 \cdot E & \ldots & 0 \\
0 & E & E & \ldots & E
\end{array}\right)^{T}
$$


where $E$ is the $n \times n$ identity matrix. Indeed, for $l, 1 \leqslant l \leqslant n$ we have

$$
\begin{aligned}
\partial_{x} g_{l} & =\partial_{x}\left(f_{l}+f_{l+n}+\ldots+f_{l+(i-1) \cdot n}\right)= \\
& =\partial_{x}\left(\partial^{i-1} e_{l}+\partial^{i-2} e_{l}+\ldots+e_{l}\right)= \\
& =\sum_{p=0}^{i-1}\left(-\sum_{q=p}^{i} \sum_{k=1}^{n}\left(\begin{array}{c}
i-p \\
q-p
\end{array}\right)\left(\partial_{t}^{q-p} a_{k l}\right) \partial^{i-1-q} e_{k}\right)= \\
& =-\sum_{p=0}^{i} \sum_{k=1}^{n}\left(\sum_{q=0}^{p}\left(\begin{array}{c}
i \\
q
\end{array}\right)\left(\partial_{t}^{q} a_{k l}\right)\right) \partial^{i-1-p} e_{k}= \\
& =-\sum_{p=0}^{i} \sum_{k=1}^{n}\left(\partial_{t}^{p} a_{k l}\right)\left(\sum_{q=p}^{i}\left(\begin{array}{c}
i-(q-p) \\
p
\end{array}\right) \partial^{i-1-q} e_{k}\right)= \\
& =-\sum_{p=0}^{i} \sum_{k=1}^{n}\left(\partial_{t}^{p} a_{k l}\right) g_{k+p \cdot n}
\end{aligned}
$$

and for all $k, 1 \leqslant k \leqslant n$, we have

$$
\begin{aligned}
\partial_{x} & \left(g_{k+p \cdot n}\right)=\partial_{x}\left(\sum_{q=p}^{i}\left(\begin{array}{c}
i-(q-p) \\
p
\end{array}\right) f_{k+q \cdot n}\right)= \\
& =\partial_{x}\left(\sum_{q=p}^{i}\left(\begin{array}{c}
i-(q-p) \\
p
\end{array}\right) \partial^{i-1-q} e_{k}\right)= \\
& =\sum_{q=p}^{i}\left(\begin{array}{c}
i-(q-p) \\
p
\end{array}\right) \partial_{x} \partial^{i-1-q} e_{k}= \\
& =-\sum_{q=p}^{i}\left(\begin{array}{c}
i-(q-p) \\
p
\end{array}\right) \sum_{l=1}^{n}\left(\partial_{t}^{q-p} a_{l k}\right) \partial^{i-1-q} e_{l}= \\
& =-\sum_{r=0}^{i-p} \sum_{l=1}^{n}\left(\begin{array}{c}
p+r \\
r
\end{array}\right)\left(\partial_{t}^{r} a_{l k}\right)\left(\sum_{s=p+r}^{i} \frac{\left(\begin{array}{c}
i-s+p+r \\
p
\end{array}\right)\left(\begin{array}{c}
i-p+p+r-s \\
r
\end{array}\right)}{\left(\begin{array}{c}
p+r \\
r
\end{array}\right)} \partial^{i-1-s} e_{l}\right)= \\
& =-\sum_{r=0}^{i-p}\left(\begin{array}{c}
p+r \\
r
\end{array}\right) \sum_{l=1}^{n}\left(\partial_{t}^{r} a_{l k}\right)\left(\sum_{s=p+r}^{i}\left(\begin{array}{c}
i-s+p+r \\
p+r
\end{array}\right) \partial^{i-1-s} e_{l}\right)= \\
& =-\sum_{r=0}^{i-p}\left(\begin{array}{c}
p+r \\
r
\end{array}\right) \sum_{l=1}^{n}\left(\partial_{t}^{r} a_{l k}\right) g_{l+(p+r) \cdot n},
\end{aligned}
$$

because

$$
\begin{aligned}
\frac{\left(\begin{array}{c}
i-s+p+r \\
p
\end{array}\right)\left(\begin{array}{c}
i-p+p+r-s \\
r
\end{array}\right)}{\left(\begin{array}{c}
p+r \\
r
\end{array}\right)} & =\frac{(i-s+p+r) !(i+r-s) ! r ! p !}{p !(i-s+r) ! r !(i-s) !(p+r) !}= \\
& =\frac{(i-s+p+r) !}{(i-s) !(p+r) !}=\left(\begin{array}{c}
i-s+p+r \\
p+r
\end{array}\right) .
\end{aligned}
$$


5.4. Covariant solution space. Fix an object $M$ of $\mathcal{M}$. Let $K$ be a parametric Picard-Vessiot extension of $\mathbf{k}$ for the equation

$$
\partial_{x} Y=A Y,
$$

where $A$ is the matrix corresponding to the $\mathbf{k}$-finite dimensional $\mathbf{k}\left[\partial_{x}\right]$-module $M$. Then the covariant solution space is

$$
V=\operatorname{ker}\left(\partial_{x}, K \otimes M\right)
$$

which is a vector space over the field $\mathbf{k}_{\mathbf{0}}$. We let

$$
V^{(i)}=\operatorname{ker}\left(\partial_{x}, K \otimes M^{(i)}\right)
$$

which is an $(i+1) \cdot \operatorname{dim} M^{(0)}=(i+1) \cdot n$-dimensional vector space over $\mathbf{k}_{\mathbf{0}}$. Let $Y \in \mathbf{G L}_{n}(K)$ be a solution matrix for $V^{(0)}$. Then, the columns of the matrix

$$
Y_{i}:=\left(\begin{array}{ccccc}
Y & 0 & 0 & \ldots & 0 \\
Y_{t} & Y & 0 & \ldots & 0 \\
Y_{t t} & Y_{t} & Y & \ldots & 0 \\
\vdots & \vdots & \vdots & \ddots & \vdots \\
Y_{t^{i-1}} & Y_{t^{i-2}} & Y_{t^{i-3}} & \ldots & 0 \\
Y_{t^{i}} & Y_{t^{i-1}} & Y_{t^{i-2}} & \ldots & Y
\end{array}\right)
$$

form a $\mathbf{k}_{\mathbf{0}}$-basis for $V^{(i)}$, since $Y_{i} \in \mathbf{G L}_{n \cdot(i+1)}(K)$. Hence, for each $M$ we have a map sending $M^{(i)} \mapsto V^{(i)}$ and $V^{(i)}$ can be identified with the vector space $\mathbf{k}_{\mathbf{0}}[\partial]_{\leqslant i} \otimes V^{(0)}$ by Proposition 5. Moreover, (5), (4), (6), and (7) are satisfied. So, there is a functor $\omega: M \mapsto V$ from the category $\mathcal{M}$ to the category $\mathcal{V}$.

\subsection{Some examples.}

Example 7. Consider a matrix $A \in \mathbf{M}_{n}\left(\mathbf{k}_{\mathbf{0}}{ }^{\left\{\partial_{t}\right\}}\right)$ and the differential equation

$$
\partial_{x} Y=A Y \text {. }
$$

Then, $V_{i}$ is the $\partial_{x}$-differential module with the matrix

$$
A_{i}=\operatorname{diag}(A, \ldots, A)
$$

and the $(i+1)$ th solution matrix is

$$
Y_{i}=\operatorname{diag}(Y, \ldots, Y) \text {. }
$$

So, we do not bring anything new to the usual differential Galois theory.

Example 8. Consider the differential equation

$$
\partial_{x} y=\frac{t}{x} y
$$


Then,

$$
\begin{aligned}
A_{0} & =\left(\frac{t}{x}\right) \\
A_{1} & =\left(\begin{array}{cc}
\frac{t}{x} & 0 \\
\frac{1}{x} & \frac{t}{x}
\end{array}\right) \\
\vdots & \\
A_{i} & =\left(\begin{array}{cccccc}
\frac{t}{x} & 0 & 0 & 0 & \ldots & 0 \\
\frac{1}{x} & \frac{t}{x} & 0 & 0 & \ldots & 0 \\
0 & \frac{2}{x} & \frac{t}{x} & 0 & \ldots & 0 \\
\vdots & \vdots & \vdots & \vdots & \ddots & \vdots \\
0 & 0 & 0 & 0 & \ldots & \frac{t}{x}
\end{array}\right)
\end{aligned}
$$

and the solution matrices are

$$
\begin{aligned}
Y_{0} & =\left(x^{t}\right), \\
Y_{1} & =\left(\begin{array}{cc}
x^{t} & 0 \\
x^{t} \log x & x^{t}
\end{array}\right), \\
& \vdots \\
Y_{i} & =\left(\begin{array}{cccccc}
x^{t} & 0 & 0 & 0 & \ldots & 0 \\
x^{t} \log x & x^{t} & 0 & 0 & \ldots & 0 \\
x^{t}(\log x)^{2} & x^{t} \log x & x^{t} & 0 & \ldots & 0 \\
\vdots & \vdots & \vdots & \vdots & \ddots & \vdots \\
x^{t}(\log x)^{i} & x^{t}(\log x)^{i-1} & x^{t}(\log x)^{i-2} & x^{t}(\log x)^{i-3} & \ldots & x^{t}
\end{array}\right),
\end{aligned}
$$

\subsection{Fiber functor.}

Proposition 6. The functor $\omega$ is an exact $\mathbf{k}_{\mathbf{0}}$-linear faithful tensor covariant functor intertwining with the functors $F^{p}$ and preserving the map $\partial$.

Proof. We have:

- Consider a short exact sequence

$$
0 \longrightarrow L \stackrel{\varphi_{1}}{\longrightarrow} M \stackrel{\varphi_{2}}{\longrightarrow} N \longrightarrow 0
$$

Since $\mathbf{k}$ is a field, the sequence

$$
0 \longrightarrow K \otimes_{k} L \stackrel{\operatorname{id}_{K} \otimes \varphi_{1}}{\longrightarrow} K \otimes_{k} M \stackrel{\operatorname{id}_{K} \otimes \varphi_{2}}{\longrightarrow} K \otimes_{k} N \longrightarrow 0
$$

is also exact. We have

$$
\begin{aligned}
\operatorname{dim}_{K} K \otimes M-\operatorname{dim}_{K} K \otimes L & =\operatorname{dim}_{K} K \otimes N \\
\operatorname{dim}_{\mathbf{k}_{\mathbf{0}}} \operatorname{ker}\left(\partial_{x}, K \otimes M\right) & =\operatorname{dim}_{K} K \otimes M, \\
\operatorname{dim}_{\mathbf{k}_{\mathbf{0}}} \operatorname{ker}\left(\partial_{x}, K \otimes L\right) & =\operatorname{dim}_{K} K \otimes L, \\
\operatorname{dim}_{\mathbf{k}_{\mathbf{0}}} \operatorname{ker}\left(\partial_{x}, K \otimes N\right) & =\operatorname{dim}_{K} K \otimes N
\end{aligned}
$$


Consider the following short sequence:

$$
\begin{array}{ccc}
0 \longrightarrow \operatorname{ker}\left(\partial_{x}, K \otimes_{\mathbf{k}} L\right) \longrightarrow \operatorname{ker}\left(\partial_{x}, K \otimes_{\mathbf{k}} M\right) \longrightarrow \\
\longrightarrow \operatorname{ker}\left(\partial_{x}, K \otimes_{\mathbf{k}} N\right) \longrightarrow 0
\end{array}
$$

The first and second elements of this sequence are subsets of the corresponding elements of the sequence (23) and, hence, sequence (24) is exact at those elements. Since $\operatorname{ker}\left(\partial_{x}, K \otimes M\right) / \operatorname{ker}\left(\partial_{x}, K \otimes L\right)$ is isomorphic to a subspace of $\operatorname{ker}\left(\partial_{x}, K \otimes N\right)$ and according to the dimensional equalities we have

$$
\operatorname{dim}_{\mathbf{k}_{0}} \operatorname{ker}\left(\partial_{x}, K \otimes M\right) / \operatorname{ker}\left(\partial_{x}, K \otimes L\right)=\operatorname{dim}_{\mathbf{k}_{0}} \operatorname{ker}\left(\partial_{x}, K \otimes N\right),
$$

the sequence (24) is exact at the third element.

- We have $\varphi=a \varphi_{1}+b \varphi_{2}$ commutes with $\partial_{x}$ for all $\varphi_{i} \in \operatorname{Hom}(M, N)$ and $a, b \in \mathbf{k}_{\mathbf{0}}$. Hence, $\varphi$ is mapped to $a \cdot \omega\left(\varphi_{1}\right)+b \cdot \omega\left(\varphi_{2}\right)$. Thus, $\omega$ is $\mathbf{k}_{\mathbf{0}}$-linear.

- A non-zero module provides a non-zero solution.

- For modules $M$ and $N$ we only need to show that

$$
\operatorname{ker}\left(\partial_{x}, K \otimes M\right) \otimes \operatorname{ker}\left(\partial_{x}, K \otimes N\right)=\operatorname{ker}\left(\partial_{x}, K \otimes M \otimes N\right),
$$

but this follows as in the usual differential case by taking $K$-bases $\left\{e_{i}\right\}$ and $\left\{f_{j}\right\}$ of $K \otimes M$ and $K \otimes N$, respectively, with $\partial_{x}\left(e_{i}\right)=\partial_{x}\left(f_{j}\right)=0$.

- Let $\varphi \in \operatorname{Hom}(M, N)$. Then it is mapped to the morphism id $\otimes \varphi$ of the spaces $\operatorname{ker}\left(\partial_{x}, K \otimes M\right)$ and $\operatorname{ker}\left(\partial_{x}, K \otimes N\right)$.

Corollary 3. The category $\mathcal{M}$ together with the functor $\omega$ is a neutral differential Tannakian category (Definition 2).

For $M \in \mathcal{O b}(\mathcal{M})$ denote by $\mathcal{C}_{M}$ the category generated by the module $M$ and all its derivatives $M^{(i)}$ and the dual $M^{*}$ using the operations $\oplus, \otimes$, and subquotient. We say that $\mathcal{C}_{M}$ is differentially generated by $M$.

Corollary 4. For a parametric linear differential equation

$$
\partial_{x} Y=A(x, t) Y
$$

a parametric differential Galois group $G$ is the functor Aut ${ }^{\otimes, \partial}(\omega)$, where $\omega: \mathcal{C}_{M} \rightarrow$ Vect $_{\mathbf{k}_{\mathbf{0}}}$ is the functor associated with $M \in \mathcal{M}$ and the action of $\partial_{x}$ defined by the matrix $A \in \mathbf{M}_{n}(\mathbf{k})$.

Proof. From Theorem 2 and Corollary 3 it follows that the category $\mathcal{C}_{M}$ together with the functor $\omega$ form a neutral differential Tannakian category. Moreover, $\mathcal{C}_{M}$ is equivalent to the category of representations of the linear differential algebraic group $\mathbf{A u t}^{\otimes, \partial}(\omega)$. It remains to show that for the parametric Galois group $G$ its category of representations is equivalent to $\mathcal{C}_{M}$.

Similar to [15, Theorem 2.33], in order to get this equivalence we notice the following. Consider a parameterized Picard-Vessiot extension $K$ of $\mathbf{k}$ corresponding to the equation $\partial_{x} Y=A Y$. This field $K$ is generated over $\mathbf{k}$ by a fundamental set of solutions of the differential equation and $G$ acts on the $\mathbf{k}_{\mathbf{0}}$-linear space generated by these solutions. So, $G$ acts on $K$ and commutes with $\partial_{x}$. Hence, if for $f \otimes m \in K \otimes M$ and $\sigma \in G$, which is an automorphism of $K$, we let

$$
\sigma(f \otimes m)=\sigma(f) \otimes m,
$$


then the linear differential algebraic group $G$ acts faithfully on $\omega(M)$, which is an object of the category $\mathcal{V}$. Indeed, recall that if one fixes a $\mathbf{k}$-basis of $M$, one gets a fundamental solution matrix, on which $\sigma$ acts by multiplication by an invertible matrix with coefficients in $\mathbf{k}_{\mathbf{0}}$.

From [17, Proposition 3] it follows that the category $\mathbf{R e p}_{G}$ is differentially generated by a faithful representation $V$ of $G$. We take $V=\omega(M)$ and, hence, for every representation $W$ there exists an object $M_{W}$ in $\mathcal{C}_{M}$ such that $\omega\left(M_{W}\right)=W$. Therefore, the functor $\omega$ is essentially surjective. We need to show that

$$
\operatorname{Hom}(M, N) \rightarrow \operatorname{Hom}(S(M), S(N))
$$

is a bijection. We have:

$$
\operatorname{Hom}_{\mathbf{k}\left[\partial_{x}\right]}(M, N)=\operatorname{ker}\left(\partial_{x}, M^{*} \otimes N\right)=\operatorname{Hom}_{\mathbf{k}\left[\partial_{x}\right]}\left(\mathbf{k}_{\mathbf{0}}, M^{*} \otimes N\right) .
$$

So, it is enough to show the bijection for $M=\mathbf{k}$. We have $\omega(\mathbf{k})=\mathbf{k}_{\mathbf{0}}$, which is the trivial representation of the group $G$. Moreover,

$$
\operatorname{Hom}_{\mathbf{k}\left[\partial_{x}\right]}\left(\mathbf{k}_{\mathbf{0}}, N\right)=\left\{n \in N \mid \partial_{x} n=0\right\}
$$

and

$$
\operatorname{Hom}_{\mathbf{k}_{\mathbf{0}}}\left(\mathbf{k}_{\mathbf{0}}, \omega(N)\right)=\left\{v \in \operatorname{ker}\left(\partial_{x}, K \otimes N\right) \mid g v=v \text { for all } g \in G\right\} .
$$

But the fixed points $K^{G}$ are $\mathbf{k}$ (see [4, Theorem 3.5]). Hence, if $g(f \otimes n)=f \otimes n$ for $f \in K$ and $n \in N$ for all $g \in G$ then $f \in \mathbf{k}$, and

$$
(K \otimes N)^{G}=N \text {. }
$$

Thus, we have shown a bijection between $\operatorname{Hom}(M, N)$ and $\operatorname{Hom}(\omega(M), \omega(N))$.

We will finally show that our more compact and, therefore, computationally friendlier way of recovering $G$ is equivalent to applying the derivation functor $F$ iteratively. Recall that if one applies $F$ twice, one multiplies the dimension by 4 . In our construction we multiply it only by 3 .

Proposition 7. For $M \in \mathcal{M}$ the category $\left(\mathcal{C}_{M}, \omega\right)$ coincides with the neutral differential Tannakian category $(\mathcal{C}, \omega)$ generated by $M$ using $\oplus, \otimes$, *, and iterated application of the first order derivation functor $F$.

Proof. First note that the category $\mathcal{C}_{M}$ is a subcategory of $\mathcal{C}$. Indeed, denote the $i$-th iteration of $F$ on $M$ by $M^{[i]}$. Then, $M^{(i)}$ can be embedded into $M^{[i]}$. For $i=1$ we have $M^{[1]}=M^{(1)}$. For simplicity, we will show an embedding $M^{(i)} \rightarrow M^{[i]}$ for $i=2$. The general case can be shown by induction on $i$. Let $m \in M$. We will then define an embedding $E$ by

$$
\begin{aligned}
1 \otimes m & \mapsto 1 \otimes 1 \otimes m, \\
\partial \otimes m & \mapsto \frac{\partial \otimes 1 \otimes m+1 \otimes \partial \otimes m}{2}, \\
\partial^{2} \otimes m & \mapsto \partial \otimes \partial \otimes m .
\end{aligned}
$$

This map is $\mathbf{k}$-linear since it preserves the degree of $\partial$. We will show that it commutes with $\partial_{x}$. Suppose a basis of $M$ is fixed and $A \in \mathbf{M}_{n}(\mathbf{k})$ defines the 
action of $\partial_{x}$ with respect to this basis. Lemma 8 provides the matrices

$$
A^{(2)}:=\left(\begin{array}{ccc}
A & 0 & 0 \\
2 A_{t} & A & 0 \\
A_{t t} & A_{t} & A
\end{array}\right) \quad \text { and } \quad A^{[2]}:=\left(\begin{array}{cccc}
A & 0 & 0 & 0 \\
A_{t} & A & 0 & 0 \\
A_{t} & 0 & A & 0 \\
A_{t t} & A_{t} & A_{t} & A
\end{array}\right)
$$

corresponding to the prolongations of $\partial_{x}$ to $M^{(2)}$ and $M^{[2]}$, respectively, from where the commutation of $\partial_{x}$ and $E$ follows. Thus, $E \in \operatorname{Hom}\left(M^{(2)}, M^{[2]}\right)$.

By Corollary 4 , the category $(\mathcal{C}, \omega)$ is a neutral differential Tannakian category and is, therefore, equivalent to $\operatorname{Rep}_{G}$, where $G$ is the parametrized differential Galois group for the module $M$. By [17, Proposition 2], the image of $\mathcal{C}_{M}$ under this equivalence coincides with the whole $\operatorname{Rep}_{G}$, and, thus, $\mathcal{C}_{M}=\mathcal{C}$.

\section{Conclusions}

Starting with a parametric linear differential equation one first constructs the parametric differential module $M$ associated with it. To apply linear algebra operations and the prolongation functor to it is the same as to do them with its solution space. In this way we construct the category $\mathcal{C}_{M}$ differentially generated by $M$ with a fiber functor $S: \mathcal{C}_{M} \rightarrow \mathcal{V}$. From this data one recovers all finite dimensional differential representations and, hence, the parametric differential Galois group of the equation.

\section{AcKnowledgements}

The author is highly grateful to his advisor Michael Singer, to Bojko Bakalov, and Daniel Bertrand for extremely helpful comments and support. Also, the author thanks Pierre Deligne, Christian Haesemeyer, Moshe Kamensky, Claudine Mitschi, Jacques Sauloy, the participants of Kolchin's Seminar in New York, Sergey Gorchinsky, and the referees for their important suggestions.

\section{REFERENCES}

[1] Braverman, A., Etingof, P., Gaitsgory, D., Quantum integrable systems and differential Galois theory, Transformation Groups 2 (1) (1997) 31-56.

[2] Cassidy, P. J., Differential Algebraic Groups, American Journal of Mathematics 94 (1972) $891-954$

[3] Cassidy, P. J., The Differential Rational Representation Algebra on a Linear Differential Algebraic Group, Journal of Algebra 37 (1975) 223-238

[4] Cassidy, P. J., Singer, M. F., Galois Theory of Parametrized Differential Equations and Linear Differential Algebraic Group, IRMA Lectures in Mathematics and Theoretical Physics 9 (2006) 113-157

[5] Hardouin, C., Structure Galoisienne des extensions itérées de modules différentiels, $\mathrm{PhD}$ thesis (2005)

[6] Hardouin, C., Hyper transcendance des systèmes aux différences diagonaux, Compositio Mathematicae 144 (3) (2008) 565-581

[7] Hardouin, C., Singer, M., Differential Galois theory of linear difference equations, Mathematische Annalen 342 (2) (2008) 333-377

[8] Hrushovsky, E., Computing the Galois group of a linear differential equation, In: Differential Galois Theory. Institute of Mathematics, Polish Academy of Sciences, Banach Center Publications 58 (2002) 97-138

[9] Deligne, P., Milne, J. S., Tannakian categories, in Hodge Cycles, Motives, and Shimura Varieties, Lecture Notes in Mathematics 900, Springer, Heidelberg (1982) 101-228

[10] Deligne, P., Catégories tannakiennes, In the Grothendieck Festschrift 2 (1990) 111-195 
[11] Kamensky, M., Differential tensor categories, (2007), preprint available online at http://www.math. uwaterloo.ca/ ${ }^{\sim}$ mkamensk/lectures/difftan.pdf

[12] Kassel, C., Quantum Groups, Springer-Verlag, New York, 2003

[13] Mac Lane, S., Categories for the working mathematician, Springer, 1998

[14] Majid, S., Foundations of Quantum Group Theory, Cambridge University Press, 1995

[15] van der Put, M., Singer, M. F., Galois Theory of Linear Differential Equations, Series: Grundlehren der mathematischen Wissenschaften, Vol. 328, Springer-Verlag, 2003

[16] Ovchinnikov, A., Differential Tannakian categories, preprint (2008) available at: http://arxiv.org/abs/0807.2497

[17] Ovchinnikov, A., Tannakian approach to linear differential algebraic groups, Transformation groups 13 (2) (2008) 413-446

[18] Saavedra, R., Catégories tannakiennes, Lecture Notes in Mathematics 265, Springer-Verlag, 1972

[19] Springer, T. A., Linear Algebraic Groups, 2nd edition, Birkhäuser, Boston, 1998

[20] Waterhouse, W. C., Introduction to Affine Group Schemes, Springer-Verlag, New York, 1979

North Carolina State University, Department of Mathematics, Box 8205, Raleigh, NC 27695-8205, USA

URL: http://www.math.uic.edu/ aiovchin/

E-mail address: aiovchin@math.uic.edu

Current address: University of Illinois at Chicago, Department of Mathematics, Statistics, and Computer Science, 851 S. Morgan Street, M/C 249, Chicago, IL 60607-7045, USA 